\title{
PRESSÃO PARCIAL DE GASES NO EXERCícIO FÍSICO
}

\author{
Carlos Eduardo Negrão*
}

\begin{abstract}
RESUMO
As grandes controvérsias existentes sobre as pressōes parciais de gases durante o exercicio físico, levam o autor a anatisar e discutir o assunto. É inicialmente considerado o que determina a pressão parcial de oxigénio e dióxido de carbono no sangue arterial e venoso. Em seguida sāo analisadas as modificaçdes que o exercicio provoca nas pressões parciais arteriais e venosas de gases. Finalmente, são sugeridos al-

guns tópicos para futuras pesquisas os quais envolvem: 1- possiveis limitaçöes pulmonares durante $o$ estorço máximo, determinando a queda $\mathrm{P}_{2} \mathrm{O}_{2}$ e suń relação com alguns mecanismos compensatórios; 2contribuição da razão $V_{A} / Q_{C}$ para a diferença entre $P_{A}$ e $P_{a}$, durante o exercício; e 3-valores de $P_{-} O_{2}$ em diferentes intensidades de exercicio e em diferentes populaçöes.
\end{abstract}

PALAVRAS-CHAVE

Pressão parcial arterial de gases, pressão parcial venosa de gases, exercício físico, ventilação alveolar, perfusão, difusāo.

"Alguma tentativa para julgar a importância da falta de oxigênio $\left(\mathrm{O}_{2}\right)$ e excesso de dióxido de carbono $\left(\mathrm{CO}_{2}\right)$ no estabelecimento e manutenção de hiperpnéia, durante o exercício físico, precisa ainda esperar mais conclusivo e detalhado conhecimento do comportamento da pressão parcial de gases na circulação arterial" (1). Embora, muitas pesquisas tenham sido feitas desde que esta opiniāo foi dada, parece que mais evidências ainda são necessárias para melhor compreender a pressāo parcial de gases durante o exercicio. A revisāo de algumas pesquisas que estudam o assunto mostram resultados conflitantes e também discórdias na explicação das trocas ocorridas na pressão parcial de gases durante o exercício.

O primeiro aspecto que precisa ser considerado sobre este assunto é o que determina a pressão parcial de oxigênio e dióxido de carbono no sangue arterial e venoso. Parece que não somente um fator, mas uma série de fatores determinam esses valores. Ventilação alveolar, perfusāo e difusão são os principais aspectos na determinação da pressão parcial de gases no sangue. Difusão está diretamente relacionada com a diferença de pressāo de gases entre os dois lados da membrana, a área de superfície e a densidade dos gases, e inversamente relacionada com a espessura da membrana alveolar. Na posição vertical, isto é, em pé a ventilação alveolar é menor no topo dos pulmōes e maior nas suas bases $(14,15) \mathrm{e}$ a mesma idéia se aplica à perfusão; contudo, o aumento em perfusão, do topo para a base, é maior que o aumento em ventilação alveolar. Durante repouso, a razão entre ventilaçāo alveolar e perfusão no topo dos pulmōes é aproximadamente 3 , enquanto que na base ela cai para 0,6. Sob estas condições e ainda ao nivel do mar, a pressāo parcial arterial de oxigênio $\left(\mathrm{P}_{\mathrm{a}} \mathrm{O}_{2}\right)$ e a pressão parcial arterial de dióxido de carbono $\left(\mathrm{P}_{\mathrm{a}} \mathrm{CO}_{2}\right)$ estão entre $90 \quad 97$ e $36 \quad 40 \mathrm{mmHg}$, respectivamente $(1,9,12,14,15,16)$. Além desses as-

Professor Assistente Doutor da disciplina de Fisiologia da EEF-USP 
pectos, "shunts" também afetam a pressão parcial de gases. "Shunt" refere-se a uma quantidade de sangue que entra no sistema arterial sem passar pelas áreas ventiladas do pulmão. Certa quantidade de sangue arterial bronquial é coletada pela veia pulmonar e ainda veias coronárias (veias Thebesianas) drenam diretamente no ventrículo esquerdo. Desta maneira, é estimado que "shunts" contribuem com $2 \%$ do total de pressão parcial de gases.

A pressão parcial venosa de gases não é somente determinada pela ventilação alveolar, perfusão e difusão, mas também pelo consumo de oxigênio e produçào de dióxido de carbono dos tecidos. Este mecanismo pode ser bem explicado pelo descarregamento da oxihemoglobina. Com o aumento do consumo de oxigênio, a hemoglobina é mais descarregada e, conseqüentemente, a pressão parcial média de oxigênio na circulação venosa $\left(\mathrm{P}_{\mathrm{v}} \mathrm{O}_{2}\right)$ diminui. Este mecanismo também altera o transporte de dióxido de carbono porque parte deste transporte depende do descarregamento de oxigênio, isto é, hemoglobina mais íon $\mathrm{H}^{+}$combina com dióxido de carbono para formar carbamino-hemoglobina. Entretanto, este mecanismo é eficiente somente na ocorrência da dissociação da oxihemoglobina. Os fenômenos mencionados acima podem ser resumidos da seguinte maneira:

$$
\begin{aligned}
& \mathrm{HbO}_{2} \longrightarrow \mathrm{Hb}+\mathrm{O}_{2} \\
& \mathrm{H}_{2} \mathrm{O}+\mathrm{CO}_{2} \longrightarrow \mathrm{H}^{+}+\mathrm{HCO}_{3} \\
& \mathrm{Hb}+\mathrm{H}^{+} \longrightarrow \mathrm{HbH} \\
& \mathrm{HbH}+\mathrm{CO}_{2} \longrightarrow \mathrm{HbCO}_{2} \mathrm{H}
\end{aligned}
$$

Além desses aspectos já mencionados, alteraçōes no consumo de oxigênio resultarão também em mudanças na produção de dióxido de carbono como produto final da fosforização oxidativa e, conseqüentemente, afetarão a pressão parcial média de dióxido de carbono na circulação venosa $\left(\mathrm{P}_{\overline{\mathrm{V}}} \mathrm{CO}_{2}\right)$, uma vez que esta pressão depende da concentração deste gás e da pressão barométrica.

O que determina a modificação da pressão parcial arterial de gases?

Muitas situaçōes contribuem para a alteração da pressāo parcial de gases. Contudo, altitude, exercício físico e submersão em água são as situaçōes mais conhecidas que afetam a pressão parcial de gases na circulação sangüínea de seres humanos. Analisando o efeito do exercício, é pertinente dividi-lo em diferentes fases para evitar exagerada simplificação, o que muitas vezes pare- ce inadequado e perigoso. No início do exercício, segundo resultados apresentados por alguns estudos $(1,2,9,17)$, a $\mathrm{P}_{\mathrm{a}} \mathrm{O}_{2}$ e $\mathrm{P}_{\mathrm{a}} \mathrm{CO}_{2}$ são pouco alteradas, isto é, são mantidas ou sofrem quedas que variam entre 5 e $10 \mathrm{mmHg}$. Todavia, esta nāo parece ser a única versão, porque outros estudos $(8,10)$ mostram que, nesta fase de exercício, a $\mathrm{P}_{\mathrm{a}} \mathrm{O}_{2}$ é aumentada e a $\mathrm{P}_{\mathrm{a}} \mathrm{CO}_{2}$ é diminuída em conseqüência do aumento da ventilação alveolar. Esta controvérsia existente parece ser explicada pela diferença de amostragem, isto é, PAN et al., 1983 estudou pôneis enquanto BARR et al., 1964, BJURSTEDT \& WIGERTZ 1971 e YOUNG \& WOOLCOCK, 1978 estudaram seres humanos. Além disso, os métodos de avaliação utilizados eram diferentes. PAN et al., 1983 aplicou testes em esteira rolante enquanto YOUNG \& WOOLCOCK, 1978 aplicaram testes em escadas, BARR et al., 1964 bicicleta em posição sentado e BJURS TEDT \& WIGERTZ, 1971 bicicleta em posição deitado. embora utilizando diferentes métodos, os estudos com seres humanos mostram concordância entre si, o que sugere que a diferença de resultados verificada em pôneis deve ser peculiar à espécie.

$O$ rápido aumento da ventilação alveolar verificado no início do exercício físico tem sido explicado por diferentes mecanismos. Contudo, dois desses mecanismos merecem ênfase. Com o intuito do exercício físico, partem impulsos do sistema nervoso central para o centro respiratório e destes, para os músculos respiratórios, o que resulta em aumento da ventilação (7). Além desse mecanismo, receptores musculares conhecidos como do tipo III e IV são extremamente sensiveis para açōes mecânicas da musculatura esquelética (7). Desta maneira, com a elevação da freqüência de contraçōes musculares (típica do exercício), esses receptores incrementam suas descargas no centro respiratório e estes, conseqüentemente, aumentam a ventilação alveolar através do aumento de descargas direcionadas nos músculos responsáveis pela respiração.

Após os três pirimeiros minutos de exercício leve ou moderado, a literatura mostra uma vez mais, resultados conflitantes pois, enquanto para alguns autores (13) a $\mathrm{P}_{\mathrm{a}} \mathrm{O}_{2}$ é mantida abaixo dos valores de repouso, para outros $(1,5,16)$ esta pressão é mantida nos mesmos níveis dos valores de repouso ou significativamente acima deles. Quanto a $\mathrm{P}_{\mathrm{a}} \mathrm{CO}_{2}$ durante o exercício leve ou moderado, parece haver concordância quanto aos resultados, isto é, esses valores permanecem próximos (levemente abaixo) daqueles achados em repouso $(8,13,16,17)$. Durante exercício pesado, a $\mathrm{P}_{\mathrm{a}} \mathrm{O}_{2}$ diminui significativamente em alguns atletas 
$(3,4,17)$. Estes resultados durante exercício pesado em atletas, parecem modificar o conceito existente da manutenção da pressão parcial arterial de oxigênio durante exercício físico. DEMPSEY et al., 1982, estudando corredores de provas longas mostrou que a $\mathrm{P}_{\mathrm{a}} \mathrm{O}_{2}$ cai até $60 \mathrm{mmHg}$ durante exercicio pesado, o que determina uma leve ou moderada dessaturação da hemoglobina para niveis de $8090 \%$. Resultados similares foram mostrados por YOUNG e WOOLCOCK, 1978, quando a $\mathrm{P}_{\mathrm{a}} \mathrm{O}_{2}$ diminui em média $33 \mathrm{mmHg}$ durante exercício submáximo.

De acordo com o esperado, a $\mathrm{PaCO}_{2}$ diminui durante o exercício máximo. A hiperventilação desenvolvida no limiar anaeróbio, em resposta ao aumento da pressão parcial venosa de dióxido de carbono e queda do $\mathrm{pH}$, diminui a $\mathrm{P}_{\mathrm{a}} \mathrm{CO}_{2}$.

Com relação à pressāo parcial arterial de gases, é também pertinente lembrar a importância da diferença da pressão parcial alveolar e arterial estabelecida durante exercício físico $(3,6,16)$. Tem sido mostrado que, durante o exercício físico, a pressão parcial alveolar de oxigênio $\left(\mathrm{PAO}_{2}\right)$ aumenta acentuadamente, enquanto a $\mathrm{P}_{\mathrm{a}} \mathrm{O}_{2}$ é mantida numa faixa que varia entre 5 e $10 \mathrm{mmHg}$ dos valores encontrados em repouso ou ainda, em alguns casos, diminuem significativamente, como nos estudos de DEMPSEY et al., 1982 e YOUNG e WOOLCOCK, 1978 citados anteriormente. Esta diferença entre pressāo parcial alveolar e capilar parece ser explicada por três mecanismos: razāo entre ventilaçāo alveolar e perfusão capilar $\left(V_{A} / Q_{C}\right)$, "shunts" e difusāo. Entretanto, a real contribuição de cada um desses fatores, durante o exercício físico, ainda não é conhecida. Para alguns pesquisadores (16), no exercício leve, a diferença de pressão alveolar e capilar diminui porque a razāo $\dot{V}_{A} / \widehat{Q}_{C}$ é mais igualmente distribuida através dos pulmões. Durante este tipo de exercício, muitos vasos sangüíneos são abertos, aumentando o fluxo de sangue nos pulmões e, conseqüentemente, diminuindo a razão $V_{A} / Q_{C}$. Para este mesmo grupo de pesquisadores, a diferença entre $\mathrm{PAO}_{2}$ e $\mathrm{P}_{2} \mathrm{O}_{2}$ somente aumenta durante o exercício moderado e máximo, quando a razão $\dot{\mathrm{V}}_{\mathrm{A}} / \hat{Q}_{\mathrm{C}}$ é ainda mais igualmente distribuída que em repouso, mas a concentração de oxigênio no sangue venoso diminui acentuadamente. Em outras palavras, a dessaturação do sangue leva à necessidade de um carregamento maior de oxigênio nos pulmōes, carregamento esse que, durante exercício moderado e máximo, não é completamente compensado pelo aumento de pressão parcial alveolar. Além desses, "shunts" também aumentam suas contribuiçōes para a dessaturação do sangue arterial. Entretanto, para outros autores uma razão mais homogênea entre $V_{A} / Q_{C}$ está longe de ocorrer. GLEDHILL et al., 1978 estudando 5 homens adultos, propôs que a razão $\dot{V}_{A} / Q_{C}$ não decresce durante o exercício físico moderado e máximo, mas sim é mantida ou aumentada. De acordo com estes autores a diferença entre $\mathrm{PAO}_{2}$ e $\mathrm{P}_{\mathrm{a}} \mathrm{O}_{2}$ aumenta devido ao não ajustamento entre $\dot{V}_{A}$ e $\hat{O}_{C}$ e também pela contribuição dos "shunts"

O que determina a modificação da pressão parcial venosa de gases?

Uma vez mais o exercício é uma das situações mais ilustrativas na modificação da pressão parcial média venosa de gases. No início do exercício, a $\mathrm{P}_{\bar{v}} \mathrm{O}_{2}$ é mantida, pois são precisos aproximadamente 30 segundos para que a $\mathrm{P}_{\bar{v}} \mathrm{O}_{2}$ diminua $(2,3)$. Este tempo de carência é devido ao aumento do consumo de oxigênio pelos tecidos em trabalho. É sabido que existe uma relação inversa entre $\mathrm{P}_{\overline{\mathrm{V}}} \mathrm{O}_{2}$ e a intensidade do exercício. Durante o exercício moderado, os valores médios do $\mathrm{P}_{\overline{\mathrm{V}}} \mathrm{O}_{2}$ estāo entre $20-25 \mathrm{mmHg}$ (13), enquanto que, em exercício máximo, estes valores podem cair para 15-20 mmHg e em alguns casos (atletas), podem chegar até entre $10-15 \mathrm{mmHg}(3)$. É interessante frisar que estes valores referem-se à pressão parcial média venosa de oxigênio $\left(\mathrm{P}_{\bar{V}} \mathrm{O}_{2}\right)$ e nāo à pressāo parcial venosa de oxigênio $\left(\mathrm{P}_{\bar{v}} \mathrm{O}_{2}\right)$ advinda dos músculos que estão em atividade. De acordo com alguns estudos $(3,9)$, a $\left(\mathrm{P}_{\bar{v}} \mathrm{O}_{2}\right)$ pode ser tāo baixa quanto $3 \mathrm{mmHg}$. contudo, este dados não parecem concordar com outros encontrados na literatura, como por exemplo os resultados de THONSOM et al., 1974 que mostram valores de 18,20 e $20 \mathrm{mmHg}$ para pressão parcial em veia femoral para exercício leve, moderado e pesado, respectivamente. Provavelmente, esta diferença de resultados é devida à seleção da amostra, isto é, DEMPSEY et al., 1982 e McARDLE et al., 1986 estudaram atletas, enquanto THONSOM et al., 1974 estudaram homens levemente treinados na faixa de 25-41 anos. O nivel de condiçāo fisica é muito importante, uma vez que condição física está diretamente relacionada à extraçāo de oxigênio nos tecidos e, portanto, inversamente relacionada à $\mathrm{P}_{\bar{v}} \mathrm{O}_{2}$. Conforme já mencionado anteriormente, os valores de $\mathrm{P}_{\bar{v}} \mathrm{O}_{2}$ são muito importantes, porque eles determinam a extensão do carregamento de oxigênio quando da passagem do sangue pelos vasos capilares pulmonares.

Contrariamente à $\mathrm{P}_{\bar{v}} \mathrm{O}_{2}$, a $\mathrm{P} \overline{\mathrm{v}} \mathrm{CO}_{2}$ é diretamente relacionada à intensidade do exercício $(9$, $13,14)$. Durante atividade aeróbia, quase que todo o dióxido de carbono é gerado pela descarbonização da glicose, enquanto que, a partir do limiar anaeróbio, uma outra importante fonte de dióxido de carbono surge, isto é, o acentuado aumento de 
ín hidrogênio é parcialmente neutralizado por bicarbonato, gerando $\mathrm{CO}_{2}$ e $\mathrm{H}_{2}$ (15).

$$
\mathrm{H}^{+}+\mathrm{HCO}_{3} \longrightarrow \mathrm{CO}_{2}+\mathrm{H}_{2} \mathrm{O}
$$

Os valores venosos para dióxido de carbono achados na literatura variam de 45 para $75 \mathrm{mmHg}$. Estes números representam desde situações de repouso até situaçōes de esforço máximo, onde existe uma somatória de dióxido de carbono expressa pela total oxidação da glicose e pela parcial compensação da acidose metabólica.

Observa-se com freqüência a simplificação das curvas de pressão parcial de oxigênio e dióxido de carbono durante esforço físico. Entretanto, se por um lado, esta simplificação pode facilitar a compreensão do comportamento destas variáveis, por outro lado pode criar certos estereótipos que nem sempre retratam o real comportamento das mesmas. Além disso, os valores achados na literatura muitas vezes não concordam entre si. Por exemplo, enquanto para alguns autores $(1,2,17)$ a $\mathrm{P}_{\mathrm{a}} \mathrm{O}_{2}$ e a $\mathrm{PaCO}_{2}$, no início do exercício, são levemente diminuidas ou mantidas, para outros a $\mathrm{P}_{\mathrm{a}} \mathrm{O}_{2}$ é aumentada (10) e a $\mathrm{P}_{\mathrm{a}} \mathrm{CO}_{2}$ é diminuída (8). Conforme já se discutiu, esta discórdia encontrada na literatura sugere a realização de mais estudos $e$ também que os resultados encontrados numa determinada espécie não devem ser generalizados para outras.

É bem sabido que a $\hat{V}_{A}$ e o volume minuto cardiaco aumentam imediatamente no início do exercício e que existem no mínimo 30 segundos de carência para que o consumo de oxigênio aumente. A partir desses conhecimentos, quedas na $\mathrm{P}_{\mathrm{a}} \mathrm{O}_{2}$ parecem inexplicáveis conforme proposto por alguns estudos $(1,2,17)$. Embora essa queda não seja norteada de grande importância, uma vez que, de acordo com a curva de dissociação de oxigênio, quedas entre $510 \mathrm{mmHg}$ abaixo dos valores de repouso (ao nivel do mar) não afetam significativamente a saturação da hemoglobina, portanto, nāo interferindo na "performance" durante exercício, mais estudos utilizando registros continuos poderiam esclarecer o que realmente ocorre nesta fase do exercício.

Considerando os resultados encontrados em algumas pesquisas, parece possível descrever 0 comportamento da $\mathrm{P}_{\mathrm{a}} \mathrm{O}_{2}$ em seres humanos da seguinte maneira: queda (5 $10 \mathrm{mmHg}$ ) no início do exercício, seguido de aumento para valores próximos daqueles encontrados em repouso. Entretanto, esta não parece ser a verdadeira situação em atletas altamente treinados quando um periodo de adaptação não é permitido antes que se atinja o esforço máximo. Conforme já mencionado, DEMPSEY et al., 1982 mostram que a $\mathrm{P}_{\mathrm{a}} \mathrm{O}_{2}$ pode sofrer drástica queda durante esforço máxi- mo em atletas quando um período de adaptação não é permitido. Segundo estes autores, três fatores podem explicar tal dessaturação do oxigênio arterial: alguns atletas não aumentam suficientemente a $\dot{V}_{A}$ para assegurar adequada oxigenação alveolar, não estabelecendo uma diferença de pressão parcial de oxigênio entre alvéolos e capilares, conhecida como gradiente de difusão; volume minuto cardíaco extremamente aumentado, verificado em atletas $\left(\begin{array}{ll}30 & 35 \\ 1 / \mathrm{min}\end{array}\right)$ durante esforço máximo, diminui o tempo de trânsito das células vermelhas do sangue nos capilares pulmonares, isto é, de 0,8 seg para 0,3 ou menos. Este mecanismo faz com que o sangue não seja completamente carregado de oxigênio durante tal esforço; finalmente, por mais paradoxal que possa parecer, o acentuado descarregamento de oxigênio verificado durante esforço máximo, e que tanto contribui para a liberação de energia na musculatura esquelética, determina um decréscimo da reserva do carregamento de oxigênio no sangue.

É conhecido através da curva de dissociaçāo de oxigênio que significantes quedas da $\mathrm{P}_{\mathrm{a}} \mathrm{O}_{2}$ diminuem a saturação de hemoglobina para valores críticos. Este fenômeno pode levar à hipóxia precoce dos músculos em atividade e, em conseqüência, uma diminuição da capacidade aeróbia. Portanto, de acordo com esta consideração, o próximo passo nos estudos de DEMPSEY et al., 1982 e YOUNG \& WOOLCOCK, 1978 será verificar em que extensão a capacidade aeróbia pode ser limitada por esta dessaturação, quais são os aspectos que podem ser relacionados para esta queda de $\mathrm{P}_{a} \mathrm{O}_{2}$ e se existem mecanismos de compensação nesses indivíduos altamente treinados.

$A$ diferença entre $\mathrm{PAO}_{2}$ e $\mathrm{P}_{2} \mathrm{O}_{2}$ durante exercício é outro assunto que merece ênfase. entretanto, para abordar tal mecanismo, nada parece mais sugestivo do que estudar o real comportamento $\mathrm{V}_{\mathrm{A}} / \mathrm{Q}_{\mathrm{C}}$ durante o exercício físico, uma vez que os resultados são um tanto quanto conflitantes (conforme já mencionado acima). "Shunts" são também reconhecidos como fatores afetando a diferença entre $\mathrm{PAO}_{2}$ e $\mathrm{P}_{\mathrm{a}} \mathrm{O}_{2}$. $\mathrm{O}$ sangue originado das artérias bronquiais contribuem para esta diferença de duas formas: durante o exercício, este sangue sofre maior dessaturação que em repouso e a quantidade de sangue originada destes "shunts" é muito maior que aquela observada em repouso. $\mathrm{O}$ mesmo mecanismo não pode ser aplicado às veias coronárias, porque elas contribuem apenas em quantidade, uma vez que as fibras miocárdias extraem aproximadamente $80 \%$ do oxigênio arterial em repouso (11). Portanto, o aumento do consumo de oxigênio durante exercício pode ser efetuado somente através da quanti- 
dade de sangue, o que torna a contribuição do "shunt" coronariano quase que desprezivel.

A pressão parcial de gases na circulação venosa parece ser muito menos estudada que na circulação arterial. Considerando que a extração de oxigênio pelas fibras musculares pode ser estimada pela $\mathrm{P}_{\bar{v}} \mathrm{O}_{2}$ e que este mecanismo contribui, em grande parte, para o uso da reserva de carregamento de oxigênio, parece que mais atençāo poderia ser dada a este assunto.

É ainda interessante lembrar que o estudo da $\mathrm{P}_{\nabla} \mathrm{O}_{2}$ é também relevante, uma vez que ele re- trata o quanto da reserva de carregamento de oxigênio é usada durante o exercício físico.

Em conclusāo, existem três principais áreas que parecem ser importantes tópicos para futuras pesquisas. Elas sāo: 1 possiveis limitaçōes pulmonares durante o esforço máximo, determinando a queda da $\mathrm{P}_{\mathrm{a}} \mathrm{O}_{2}$ e sua relação com alguns mecanismos compensatórios: 2- a real contribuição da razāo $V_{A} / O_{C}$ para a diferença entre $P_{A} e$ $\mathrm{P}_{\mathrm{a}}$, durante exercício; e 3-valores de $\mathrm{P}_{\bar{v}} \mathrm{O}_{2} \mathrm{em}$ diferentes intensidades de exercicio e em diferentes populaçōes.

\section{ABSTRACT:}

The controversies about gas partial pressures during exercise bring the athor to analyse and discuss that subject. Initially, the paper takes into account the mechamisms which establish the oxygen and carbon dioxide partial pressures in the arterial and venous circulation. There after, the author analyses the modifications that exercise causes in the arterial and venous partial pressures. Finally, the author suggests some topica for future researchs which involve: 1 - possible pulmonar limitations during maximal exercise, which determine falls in $\mathrm{PaO}_{2}$ and its relationship to some compensatory mechanisms; 2 - the contribution of the $V_{A} / Q_{C}$ ratio for the $P_{A}$ and $P_{a}$ difference during exercise and 3. values for $\mathrm{P}_{\mathrm{V}} \mathrm{O}_{2}$ in different intensities of exercise and in different populations.

\section{REFERÊNCIAS BIBLIOGRÁFICAS}

1 BARR, P.O. et alii. Time of blood gas changes proposed by light and moderate exercise in man. Acta Physiologica Scand., $60: 1$-17. 1964.

2 BJURSTEDT H. \& WIGERTZ, O. Dynamics of arterial oxygen tension in response to simusoidal work load in man. Acta Physiologica Sacand., 82: 236-49, 1971.

3 DEMPSEY J.A. et alii. Limitations to exercise capacity and endurance: pulmonary system. Canadian Journal of Apllied Sport Science, 7: 4-13, 1982.

4 DEMPSEY J.A. Is the lung built for exercise? Medicine and Science in Sports and Exercise, 2: 143-54, 1986.

5 GIMENEZ, M. et alii. Square-ware endurance exercise test (SWEET) for training and assessment in trained and untrained subjects. European Journal of Applied Physiology, 49: 369-77, 1982.
6 GLEDHILL, N. et alii. $V_{a} / Q_{c}$ inhomogeneity and $A-\mathrm{VO}_{2}$ difference in man during exercise: effect of $\mathrm{SF}_{6}$ breathing. Journal of Applied Physiology, 45: 512-15, 1978.

7 GUITON, A.C. Textbook of Medical Physiology. Philadelphia, W.B. Saunders, 1986.

8 HANSON, P. et alii. Determinants and consequences of ventilatory responses to competitive endurance running. Journal of Applied Physiology, 52: 615-23, 1982.

9 McARDLE, W.D. et alii. Exercise Physiology: energy, nutricion, and human performance, Philadelphia, Lea S. Febiger, 1986.

10 PAN, L.G. et alii. Hyperventilation in ponies at the onset of and during steady-state exercise. Journal of Applied Physiology, 54: 1394-402, 1983. 
11 RICHALET J.P et alii. Myocardial oxygen extraction and oxygen-hemoglobin equilibrium curve during moderate exercise. Europen Journal Applied Physiology, 47: 27-29, 1981.

12 - SHEPARD, R.H. Effect of pulmonary diffusing capacity on exercise tolerance. Journal of Applied Physiology. 12: 487-8, 1958.

13 THONSON, J.M. et alii. Oxigen transport and oxyhemoglobin dissociation during prolonged muscular work. Journal of Applied Physiology, 37: 658-64, 1974.
14 THONSON, J.M. et alii. $\mathrm{O}_{2}$ transport during exercise following blood reinfusion. Journal of Applied Physiology, 53: 1213-19, 1982.

15 WEST J.B. Respiratory physiology - the àssentials. Baltimore, Williams and Wilkins, 1982.

16 WHIPP, B.J. \& WASSERMAN, K. Alveolararterial gas tension differences during graded exercise. Journal of Applied Physiology, 27: 361-65, 1969.

17 YOUNG, I.H. \& WOOLCOCK, J. Changes in 1:!: ial blood ga? tensions during unsteady-state exercise. Journal of Applied Physiology, 44: 93-6, 1978. 